Volume 10, No.3, May - June 2021

International Journal of Advanced Trends in Computer Science and Engineering

Available Online at http://www.warse.org/IJATCSE/static/pdf/file/ijatcse1301032021.pdf

https://doi.org/10.30534/ijatcse/2021/1311032021

\title{
Pizza Dough Service Provider at Various Area Followed by Depth-First Search Algorithm
}

\author{
Ambreen $^{1,2}$, Dr Kausar Qureshi ${ }^{3}$, Dure Shahwar ${ }^{4}$ \\ ${ }^{1}$ Sindh Madrassatul Islam University Karachi Pakistan, Ambreen.khan@bbsul.edu.pk \\ ${ }^{2}$ Benazir Bhutto Shaheed University LyariKarachi Pakistan, Ambreen.khan@ bbsul.edu.pk \\ ${ }^{3}$ University of Stirling UK, kausar.qureshi@ stir.ac.uk \\ Bahria University Karachi, Pakistan, nisha.bapar11@gmail.com
}

\begin{abstract}
To figure out process that attend time investment in terms of material delivery at the nearest points is always a challenging. So that people globally finds easiest way to reach their destinations within time. Moreover the edge relatedness regulate the minimum number of paths among dealer and receiver. Here the confer question is belonging to the pizza ingredients delivery time at privileged areas. The number of branches which are located at discrete spot and are challenging with reference to time, the proposed methodology will aid the legitimate string of branches by using depth first search algorithm which will produce the optimum results at its deeper expands. The proposed graph reflects all the possible routes on which a rider can go for timely delivery of pizza doughon the basis of minimum path it has estimated.
\end{abstract}

Keywords: Pizza Service, Depth First Search, Genetic Algorithm, Permutation rules

\section{1- INTRODUCTION}

Food is an elemental precondition for every individual, and the flavored food is craving especially when it comes to Pizza. Pizza is an [4] Italian luscious dish topped with various ingredients, and dough is the basic layer on which the whole material relies. Different brands chooses different dough making factory [6]from where the dough becomes ready like Pizza Max, Pizza Hut, Pizza Crust, California Pizza, Broadway Domino's Pizza and many more, the one which is processed under our research is Pizza hut, which is more promising brand[7] for the taste of Italian dish, each of branch is dealing more than 600 pizza per day which is colossal quantity for any running branch, this is how each of branch for Pizza Hut is focused for time management, the company which provides dough throughout branches.

\subsection{Search Algorithm}

Searching Algorithms are devised to check and retrieve[2] for an elements which is wellstudied task determined by search problems stored in data structure or computed within discussed problem of any form either discrete or continuous values.

\subsection{Informed Search Algorithm}

Informed Search Algorithms endorse the prior knowledge for attaining the goal state, furthermore the fewer [6] search space takes place and finds goal node efficiently. This division of search algorithm is more useful for large search space so we labelled them heuristic. This heuristic is basically a function which works for finding the most auspicious paths. As it takes the initial node as an input and leads further to get goal estimation.

\subsection{Uninformed Search algorithm}

It is a general concept of search Algorithms which promote operations in "Brute Force Way". Uninformed Search Algorithms work without preserving any definitive intelligence of their domain or simply they don't know how to traverse tree so we termed them blind search.

\section{2- RELATED WORK}

In the literature, the most relevant work in this domain is carried by [1] the main tool for minimal path set is used for network measuring which searches for ordered node subsets with time complexity $O\left(n e^{\mathrm{n}}\right)$, where $\mathrm{n}$ is taken as total number of nodes and the problem is solved by Depth First Search (DFS) Algorithm likewise [2] Automated Teller Machine (ATM)services for immediate response from banking, the said problem focused "Padang Sidempuan" city which has multiple "ATMs" in various places thus researcher followed Depth First Search (DFS) Algorithm in comparison with Greedy Algorithm[1] for searching nearer Automated Teller Machine (ATM) from current location. The main motive of Researcher is to apply two different algorithms on same problem and compare the results achieved by proposed techniques. Hence the result were similar for both applied algorithms Depth First Search and Greedy Algorithm in terms of complexity so the acquired average running time[7] for Depth First 
Search is 239.9675 milliseconds with average distance 3033.555 meters and average running time for Greedy Algorithm 274.8501 milliseconds and the average distance is 2035.2568 meters.Therefore an analyst concluded that Depth First Search (DFS) is more efficient than Greedy Algorithm in running time though Greedy Algorithm generates better shorter distance than Depth First Search (DFS).The work anticipated by researcher [3] based on network edge connectivity where node synchronization is not dealt for proposed algorithm Depth First Search (DFS), therefore methodology focuses on available edges within tree and root node start as a distributed Depth First Search (DFS) moreover the results got from proposed technique are $48 \%$ for an average accuracy ratio.Another work in this field is carried by [4] whose objective were to develop "fast algorithm of graph coloring" which give vertices sequence in the graph followed by Depth First Search (DFS). To implement this idea researcher has [12]used "large and small benchmark graphs" and applied four different coloring algorithms like BC-COL Algorithm, DSATUR algorithm, A cutting plane Algorithm and new DSATUR algorithm.Wamiliana et-al.[8] recommended work for minimum spanning tree problem followed by Depth First Search (DFS)and Kruskal's Algorithm where they have directed for[10] total minimum connection sin weighted graph and its maintenance for maximum number of edges for each vertex. Researcher exhibit the work from the base of Minimum Spanning Tree and had applied restrictions on each of the vertex. Hence connection [5] can be poor with involvement of few factors like fund and weather.

\section{3- METHODOLOGY}

\subsection{Shortest Path:}

Shortest path (SP) is an intelligent way to find optimum routes between nodes in the graph, here the confer problem is directing routes among facilitator which is dough making company for pizza making and its delivery to the nearest branches, the basic goal to achieve such targets we have focused on the

The above figure depicts the movement of all paths from one destination to the next here supplier is the main source from which dough is delivered to all branches of Pizza Hut in Karachi, Pakistan. The number of branches located in Gulshan District are 20 which are receiving dough services from company on daily basis. Supplier inclined to deliver dough at technique of Depth First Search, Depth first Search searches the solution till the last node it has for any suggested problem.

\subsection{Depth First Search}

To figure out the working of depth first search, it expands the nodes as deep as the solution found or simply the knot which doesn't expand to anymore children. Here, if any of the node is not visited then the tree may traverse to check for each possible node. The said problem can be solved by the technique Depth First Search (DFS) $P$ is called as the abbreviation for Pizza which is locating its nearby branches for early and timely delivery of ingredients like pizza dough (PD), where the initial node Is taken as P0, from where the tree started to find the optimum solution, and the total number of targeted pizza hut branches are 20 at first phase which cover Gulshan district $P 1$ is taken as the first node from where the graph will start finding pathsand $P 2$ is the step which will be taken for new node, when cursor locates for the next node while station at $P 2$ and chooses which is the next path similarly each of possible nodes will be visited until all 20 nodes are visited properly in sequence

$$
\begin{aligned}
P 1 \rightarrow P 2 \rightarrow P 3 & \rightarrow P 4 \rightarrow P 5 \rightarrow P 6 \rightarrow P 7 \rightarrow P 8 \\
& \rightarrow P 9 \rightarrow P 10 \\
P 11 \rightarrow P 12 \rightarrow P 13 \rightarrow P 14 \rightarrow P 15 \rightarrow P 16 \rightarrow P 17 & \rightarrow P 18 \rightarrow P 19 \rightarrow P 20
\end{aligned}
$$

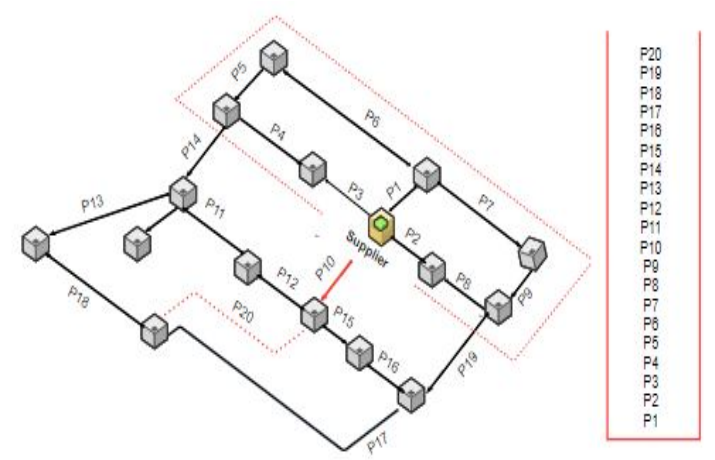

Figure 1Schema represents minimum possible paths

minimum time to all existing Pizza Hut branches in Gulshan district, however the graph shows the possible routes which needs to be visited once to check the distance moreover stack displays the number of visited noted from $P 1$ till the last node $P 20$ the node which is visited last it will be removed first from stack, similarly all nodes will be removed from stack. 


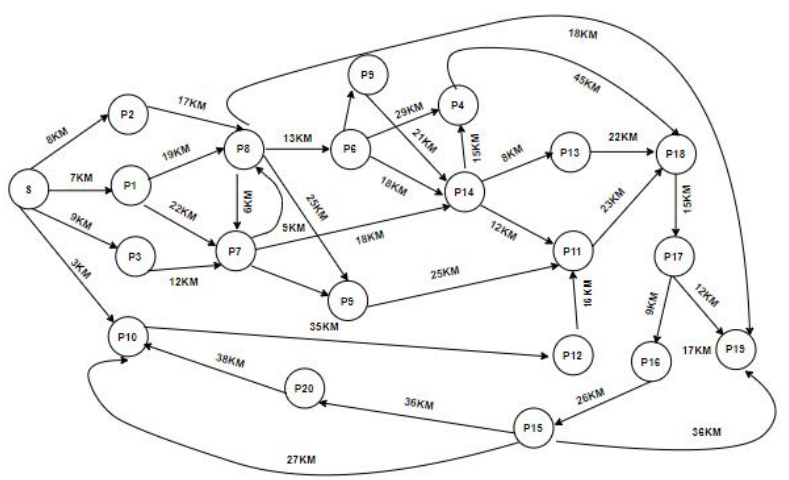

Figure 2Measured distance for Pizza Hut branches

Distance is an analytical measurement which marks how far two points are, here distance is calculated from Google Map for each node which will be beneficial to summarize paths with weights they carrying. Different weights show the shortest and longest route from supplier and will be arranged in a new sequence

\subsection{Path performance}

The quick paths which exit from the main location are considered as minimum path from suppliers so the representation of path can be illuminated for first near paths

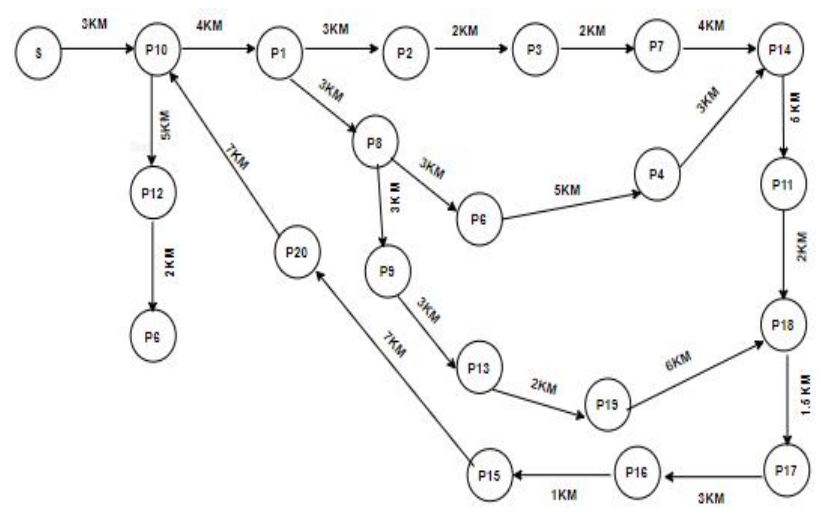

Figure 3Graph depicts the visited routes

Depth first search is combinatorial [13] for path performance on classical crossover for one point, two point, and uniform for mapped orders. To choose nearest points on the basis of distance the best path is selected with least distance value. The number of targeted pizza branches are 20 , whose distance is measured from Google map manually from point to point as

$$
\begin{gathered}
S=\left(\begin{array}{lllll}
10 & 1 & 2 & 3 & 7
\end{array}\right), \\
S=\left(\begin{array}{lllll}
4 & 1 & 14 & 17 & 16
\end{array}\right), \\
S=\left(\begin{array}{lllll}
15 & 20 & 5 & 12 & 6
\end{array}\right), \\
S=\left(\begin{array}{lllll}
8 & 9 & 13 & 19 & 18
\end{array}\right) .
\end{gathered}
$$

The mentioned paths show the sequencing of route from base point and the first visited node will be depending on its best minimum distance here in the situation, we can segregate them through ("I") mark.

$$
\begin{aligned}
& S=\left(\begin{array}{lllll}
10 & 1 & \mid & 3 & 7
\end{array}\right), \\
& S=\left(\begin{array}{llllll}
4 & 1 & 14 & 17 & 16
\end{array}\right), \\
& S=\left(\begin{array}{llllll}
15 & 20 & 5 & 12 & 6
\end{array}\right), \\
& S=\left(\begin{array}{llllll}
8 & 9 & \mid & 13 & 19 & 18
\end{array}\right) .
\end{aligned}
$$

This way, we can assume the initial values as visited and rest has to be visited at minimal path.

$$
\begin{gathered}
S=\left(\times x \mid \begin{array}{lll}
2 & 3 & 7
\end{array}\right), \\
S=\left(\times x \mid \begin{array}{llll}
14 & 17 & 16
\end{array}\right), \\
S=\left(\times x \mid \begin{array}{llll}
5 & 12 & 6
\end{array}\right), \\
S=\left(\times \times \mid \begin{array}{llll}
13 & 19 & 18
\end{array}\right) .
\end{gathered}
$$

Hence the visited routes will not be revisited for better performance of path, here we will be taking next values to check their paths and will mark them by " $\times$ "

$$
\begin{aligned}
& S=(\times \times \times \times \mid 7), \\
& S=(\times \times \times \times \mid 16), \\
& S=(\times \times \times \times \mid 6), \\
& S=(\times \times \times \times \mid 18) .
\end{aligned}
$$

The possible paths are vacating for minimal path at possible routes, thus the last meeting points

$(7,16,6,18)$ will be called at last rotation of dough delivery. This way, we may calculate the shortest distance at each point by using depth first search algorithm. The final vision of equation will represent as

$$
\begin{aligned}
& S=(\times \times \times \times \times), \\
& S=(\times \times \times \times \times), \\
& S=(\times \times \times \times \times), \\
& S=(\times \times \times \times \times) .
\end{aligned}
$$

All routes for 20 mentioned branches are visited and hence found the nominal paths ordering from supplier to its last node "20" 


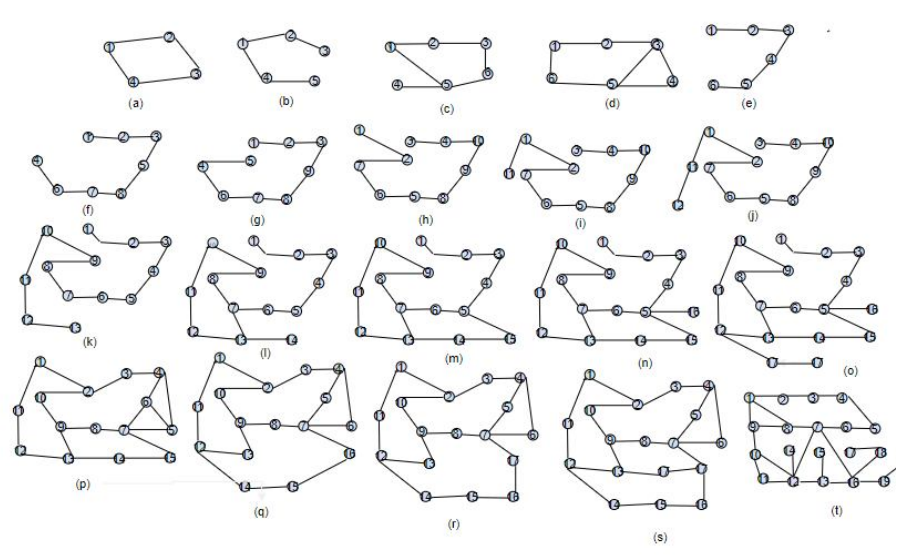

Figure 4All possible paths for pizza dough delivery

\section{4- RESULTS}

We have used genetic algorithm to propose path represestation, where we have targeted 20 branches of Karachi Pakistan and have calculated distance among each points, on the basis of distance measure from Google map we have concluded the best path for all routes from supplier to discussed 20 number of branches for their nodes and edge counts.

The transition table represents the distances measured from map and have eliminated those paths which were visited once. The representation of numbers from 1 till 5 is basically group of branches who are near to each other from base location.

Table 1 Transition table for pizza branches

\begin{tabular}{lccccc}
\hline Branch & Distance & 1 & 2 & 3 & 4 \\
\hline P01 & $7 K M$ & 10 & 1 & 2 & 3 \\
P02 & $5 K M$ & 4 & 1 & 14 & 17 \\
P03 & $6 K M$ & 15 & 20 & 5 & 12 \\
P04 & $3 K M$ & 8 & 9 & 13 & 19 \\
P05 & $4 K M$ & $\times$ & $\times$ & 2 & 3 \\
P06 & $2 K M$ & $\times$ & $\times$ & 14 & 17 \\
P07 & $16 M$ & $\times$ & $\times$ & 5 & 12 \\
P08 & $3 K M$ & $\times$ & $\times$ & 13 & 19 \\
P08 & $5 K M$ & $\times$ & $\times$ & $\times$ & $\times$ \\
P10 & $5 K M$ & $\times$ & $\times$ & $\times$ & $\times$ \\
P11 & $6 K M$ & $\times$ & $\times$ & $\times$ & $\times$ \\
P12 & $6 K M$ & $\times$ & $\times$ & $\times$ & $\times$ \\
P13 & $7 K M$ & $\times$ & $\times$ & $\times$ & $\times$ \\
P14 & $7 K M$ & $\times$ & $\times$ & $\times$ & $\times$ \\
P15 & $16 M$ & $\times$ & $\times$ & $\times$ & $\times$ \\
P16 & $3 K M$ & $\times$ & $\times$ & $\times$ & $\times$ \\
P17 & $2 K M$ & $\times$ & $\times$ & $\times$ & $\times$ \\
P18 & $5 K M$ & $\times$ & $\times$ & $\times$ & $\times$ \\
P19 & $3 K M$ & $\times$ & $\times$ & $\times$ & $\times$ \\
P20 & $5 K M$ & $\times$ & $\times$ & $\times$ & $\times$ \\
\hline
\end{tabular}

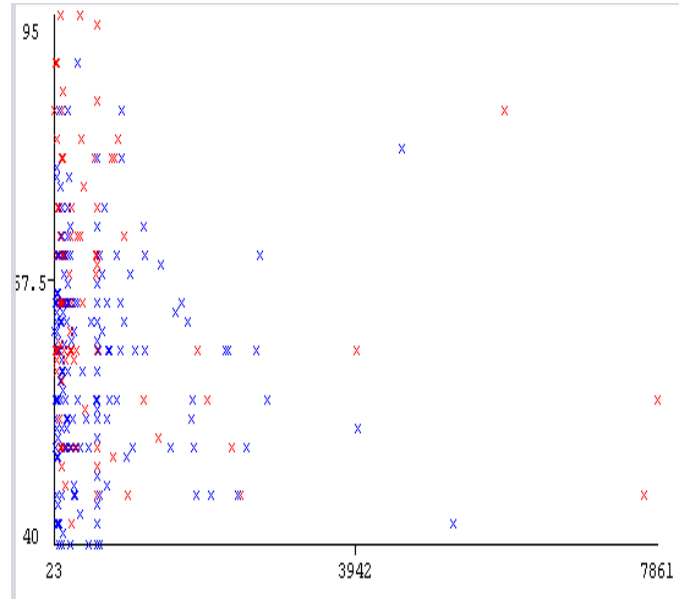

Figure 5 Optimal path using DFS(10 branches)

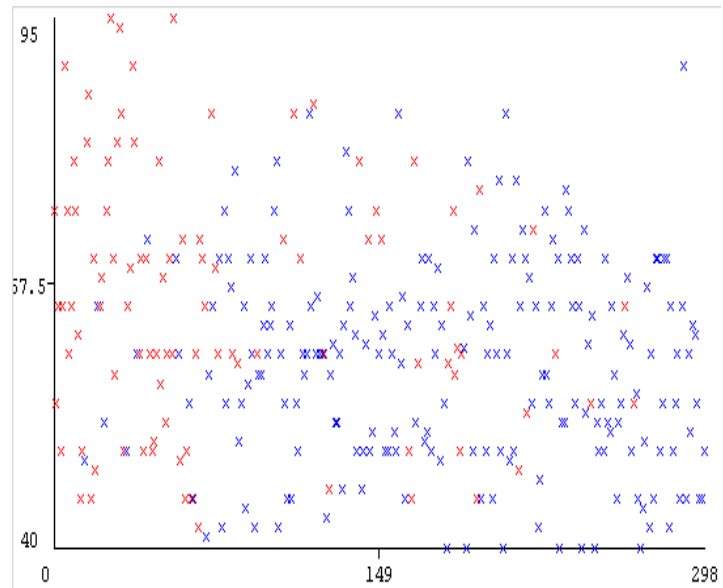

Figure 6 Optimal path using DFS (20 branches)

\section{5- CONCLUSION AND FUTURE WORK}

This work presents the efficient concept for finding the minimum path. This method is implemented on finding all possible paths with time complexity $\mathrm{O}(\mathrm{ne})$ on ordered nodes and subsets. Depth first search algorithm works for finding the nodes which connect to the route as it may not obtained the optimal solution always. Even if the path is find at the first child node we can conclude it with the optimal solution for any path relating problem. Hence the dependency occur on the first node. The extensive experiments on several problems are representing through figure 4 which actually depicts the all possible paths on benchmark network. The proposed algorithm is capable to hold and work on such problems like path finding, minimum pathor suitable path in efficient time. In addition, we can extend the work on such benchmark dataset to acquire more efficient results on benchmark problems as well. 


\section{ACKNOWLEDGMENT}

I would like to prompt my gratitude to my course Instructor and supervisor, Prof.DrAftab Ahmed Shaikh who guided, supported and offered me deep insight into the study.

\section{REFERENCES}

1. Wenbo Zhu and Wei-Chang Yeh, Senior Member, IEEE et al "A New Node-based Concept for Solving the Minimal Path Problem in General networks,"

2. Dian Rachmawati and Syahril Effendi et al, "Comparative analysis of depth first search algorithm and greedy algorithm at nearest atm search in padangsidepmuan city", Journal of Theoretical and Applied Information Technology 15th September 2020. Vol.98. No 17

3. OnurUgurlu and VahidKhalilpourAkram et al "A Distributed Depth First Search based Algorithm for Edge Connectivity Estimation," Published in: 2020 16th International Conference on Network and Service Management (CNSM)

4. Sumit Gupta and DhirendraPratapSingh "Greedy"Graph Coloring Algorithm Based on Depth First Search” International Journal on Emerging Technologies

5. Ariel FelnerRoni Stern Solomon EyalShimony and Eli Boyarski et al "Search-Based Optimal Solvers for the Multi-Agent Pathfinding Problem: Summary and Challenges,"

6. "Autonomous delivery robots and their potential impacts on urban freight energy consumption and emissions" MigueFigliozzia and Dylan Jenningsa Published by Elsevier

7. M. ErolGenevois and D. Celik, H. Z. Ulukan "ATM Location Problem and Cash Management in Automated Teller Machines"World Academy of Science, Engineering and Technology International Journal of Industrial and Manufacturing Engineering Vol:9, No:7, 2015

8. Wamiliana and Mustofa Usman et al "The Hybrid of Depth First Search Technique and Kruskal's Algorithm for Solving The Multiperiod Degree Constrained Minimum Spanning Tree Problem"Published in 2015 4th International Conference on Interactive Digital Media (ICIDM)

9. SharleeClimer and Weixiong Zhang "Cut-and-solve: An iterative search strategy for combinatorial optimization problems" Artificial Intelligence Volume 170, Issues 8-9, June 2006, Pages 714-738

10. Xifeng Yan Jiawei Han "gSpan: Graph-Based Substructure Pattern Mining" Published in 2002 IEEE International Conference on Data Mining, 2002.

11. Nisa K S "H-GRAPH: A DATA STRUCTURE FOR RETRIEVING SHORTEST PATH FROM WEIGHTED GRAPHS IN CONSTANT TIME"
12. Rakefet Ackerman and Elad Yom-Tov et al "Using confidence and consensuality to predict time invested in problem solving and in real-life web searching" 